\title{
Challenges of Sarcasm Detection for Social Network: a Literature Review
}

\author{
Afiyati Afiyati', Azhari Azhari², Anny Kartika Sari ${ }^{3}$, Abdul Karim ${ }^{4}$ \\ ${ }^{I}$ Teknik Informatika- Fakultas Ilmu Komputer - Universitas Mercu Buana, Indonesia \\ ${ }^{1,2,3}$ DIKE/FMIPA - Universitas Gadjah Mada, Indonesia \\ ${ }^{4}$ Business Administration \& Economics - National College Rahim Yar Khan, Pakistan \\ 1afiyati.reno@mercubuana.ac.id
}

\begin{abstract}
Nowadays, sarcasm recognition and detection simplified with various domains knowledge, among others, computer science, social science, psychology, mathematics, and many more. This article aims to explain trends in sentiment analysis especially sarcasm detection in last ten years and its direction in the future. We review journals with title's keyword "sarcasm" and published from year 2008 until 2018. The articles were classified based on the most frequently discussed topics among others: the dataset, pre-processing, annotations, approaches, features, context, and methods used. The significant increase in the number of articles on "sarcasm" in recent years indicates that research in this area still has enormous opportunities. The research about "sarcasm" also became very interesting because only a few researchers offer solutions for unstructured language. Some hybrid approaches using classification and feature extraction are used to identify the sarcasm sentence using deep learning models. This article will provide a further explanation of the most widely used algorithms for sarcasm detection with object social media. At the end of this article also shown that the critical aspect of research on sarcasm sentence that could be done in the future is dataset usage with various languages that cover unstructured data problem with contextual information will effectively detect sarcasm sentence and will improve the existing performance.
\end{abstract}

Keywords: sarcasm, recognition, detection, classification, performance

\section{INTRODUCTION}

According to Big Indonesian Dictionary (Kamus Besar Bahasa Indonesia), sarcasm is an expression or style of utterance that has the opposite meaning from the written words in order to insinuate, ridicule, or offend someone, a product, or an institution. A research found that sarcasm consists of components such as a speaker that conveys the utterance for the listener with a certain context that has the meaning of literal proposition but expected to be understood differently or intended scheme [1]. In other words, sarcasm is a sentence that has the opposite meaning from the written words or spoken words. Because of this different meaning, sarcasm sentences are not easily understood by the reader or hearer. Examples of sarcastic sentences are as follows: "I ordered an item A, but they sent an item B. What an excellent service!". Although there are many positive words and praise words at the end of the sentence, this sentence is a harsh sentence that shows the disappointment of a buyer who orders an item but gets a different piece from what he desired. Irony can be perceived as a result of incongruity between the context and the statement [2], [3], [4]. Meanwhile, sarcasm is also found as a result of incongruity between the context and the statement but more often intended to mention, mock or offend someone, a product or an institution [5], [6].

Every country in the world has its language style, structure, and the rules of the sarcasm sentence as well. Sarcasm detection can be as essential as sentiment analysis, especially for texts written on social media. The most sarcasm detection studies can be found in India, USA, UK, and a few studies have been found using Indonesian language texts. One sarcasm detection study that used Indonesian-language, by using the SVM, Naive Bayes, and Maximum Entropy methods, only got an accuracy value that was no greater than $60 \%$ [7]. Though the detection of sarcasm will be needed among public figures, political events, producers of a product, or an organization that uses social media as their communication application with their constituents, customers, or organizations, producer4

The difficulty in understanding sarcastic sentences could cause problems with the natural language processing concepts for online news reviews, politician dialogue sentences, institution website, or product monitoring feedback [8]. The most widely used method for detecting sarcasm has been reviewed by Wicana et al. [9]. Several other researchers also explored the part of sarcasm, such as the theory of sarcasm itself, the nature of syntax, psycholinguistics from sarcasm, lexical 
features, the nature of semantics, and others. This literature research will focus on figurative sentences as part of the sentiment analysis area, which is currently widely written on social media, online discussions, and news forums. The aim of this article is to show most commonly approaches to detect sarcastic sentences and examine published literature to provide insights for future sarcasm detection systems to practitioners and researchers.

\section{METHODS}

Explaining research chronological, including research design, research procedure, in the form of algorithms, Pseudocode, or other, and how to test and data acquisition. The description of the course of research should be supported references, so the explanation can be accepted scientifically. Research of sarcasm detection are scattered across journals of various disciplines such as social sciences, computer science, psychology medicine, etc. The journal articles and conference articles that we collected in this research taken from the Scopus website (https://www.scopus.com). The articles were obtained using a keyword "sarcasm" and were limited to the area of Computer Science. The following section will explain the procedures performed in extracting the articles used for this literature review study.

\section{A. Data Resources and Filtering Procedures}

We started outreaching the articles for this literature study from the publication database website, Scopus, since this site is one of the databases used by most researchers. We took all the articles published from 2008 until 2018, with the keyword "sarcasm." However, in 2018 and above, the research on this subject became a trending topic, and the number of articles increased sharply. Table I shows the article filtering criterias that are used in this review.

For the first filtering, articles were selected if they met the keyword "sarcasm," and we found 832 items from the Scopus website. In the second stage of the filtering, we found 670 documents limited only for journal and conference articles from practitioners and academics often use journals and conferences to acquire and publish research discovery. For our third stage of the document selection process, we selected only documents originating from the field of research in computer science. From this filtering stage, 230 articles were obtained and used for this literature review. Finally, on the fourth filtering, we select the articles that published only since the year 2008 until 2018 to limit the screening. The last screening process is selecting the articles using a keyword "sarcasm" that contained titles, abstracts, keywords, and conclusions and found 68 articles as the final documents to study in this review. Thus, as the last process, we analyzed the contents of each document based on a predetermined classification scheme that will be explained in the next section. Fig.1 shows the sequence of the process that we used to filter the articles that met all specified criteria.

\section{B. Statistical Analysis}

From the documents screening stages, it is shown that research on sarcasm detection was mostly carried out by researchers from the field of computer science, and then followed by social sciences, art and humanities, mathematics, neuroscience, engineering, medicine, business management, and others. This fact is shown in Fig. 2. Computer science researchers may write computer algorithms that mathematically shows what peoples write on social media. The algorithm enables the detection of sarcastic statement by using a combination of some features and recognize the sarcasm sentences from some variation of platforms such as online news, Twitter, Instagram, or Tumblr.

TABLE I

ARTICLE FILTERING CRITERIAS

\begin{tabular}{ll}
\hline \multicolumn{1}{c}{ Criteria } & \multicolumn{1}{c}{ Description } \\
\hline Time frame & 2008 - 2018 \\
Keywords & "Sarcasm" \\
Document type & Journal article and conference \\
Search in & Article title and abstract and \\
Search databases & SCOPUS research database \\
Type of & Peer-reviewed \\
Field & Computer science \\
\hline
\end{tabular}




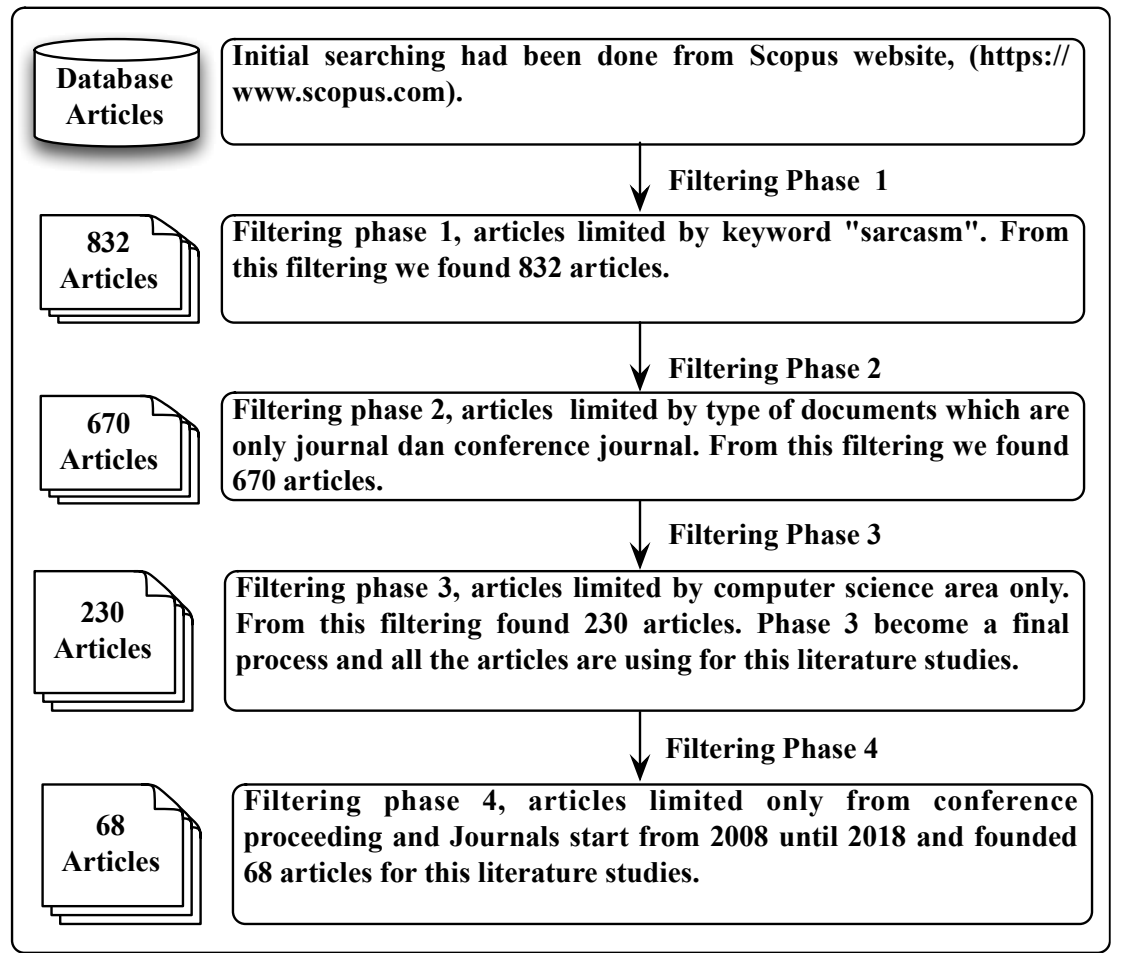

Fig. 1 Articles filtering procedure

The distribution of articles with a total of 68 articles written by researchers from 2008 to 2018 taken from the Scopus website, with the search keyword "sarcasm," type of articles is restricted to journal and conference articles, and the area of research is in computer science. Meanwhile Fig. 3 shows that the number of studies has increased significantly over the last five years. The dataset used by these studies were taken from social media, such as Twitter, WhatsApp, Amazon's sales media, and news media. The most productive country that has been doing sarcasm research, namely India and followed by the USA, Spain, and the UK. Sarcasm detection on Indian languages is one of the most challenging tasks of Natural Language Processing because Indian words are ambiguous in nature and rich in morphology [10].

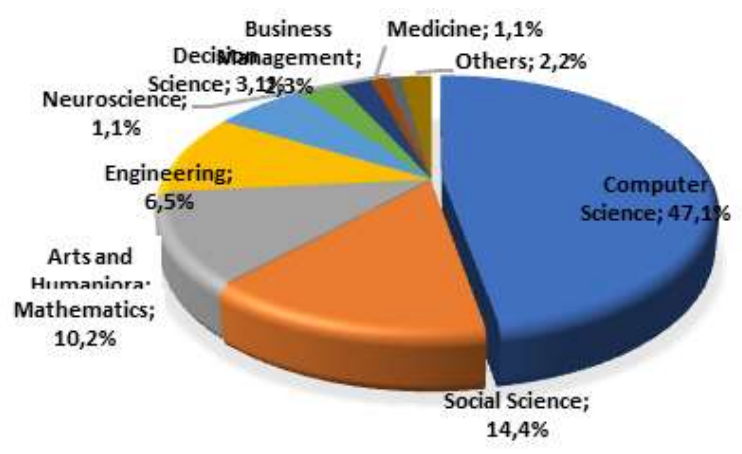

Fig. 2 Statistical analysis of articles filtering procedure



Fig. 3 Increasing of sarcasm research by year

\section{Result And Discussion}

To be more systematic in expressing research on sarcasm where a study of classification outline was expanded. This scheme of classification was categorized with focuses on 68 chosen articles from filtration processes, as we have seen in Fig. 4. 


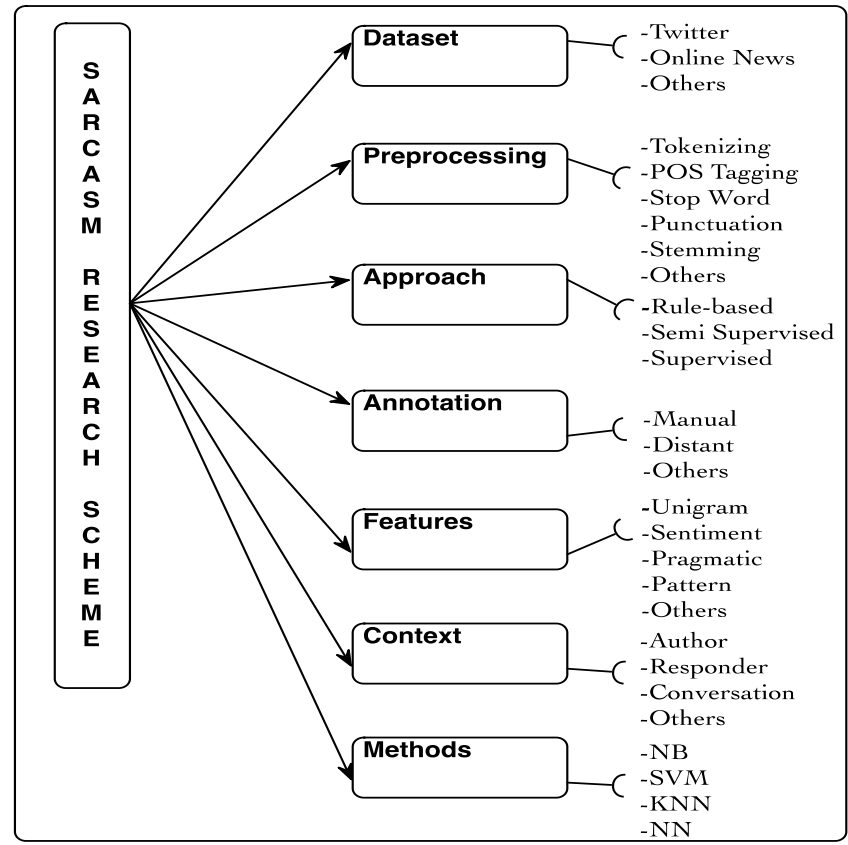

Fig. 4 Classification scheme of sarcasm research

We grouped the all articles into several schemes, which were; the dataset, the pre-processing, the approaches, the annotations, the features, the context, and the methods as we can see in Table II with an explanation of each scheme as follow:

1) Dataset: The most employed dataset that studies by many researchers are Twitter because of its distinguishable properties comparing to another kind of dataset. Twitter data contain various kinds of characters, texts, or emoticon, and it is essential to process the data before applying to feature extraction. Data preprocessing includes cleaning, tokenization, POS-tagging, stop word, punctuation, stemming, etc. [11]. Other datasets that are not widely used by a researcher are online news and WhatsApp group [10], [12].

2) Pre-Processing: The best classifiers are chosen and paired with various pre-processing provide the best possible accuracy [13]. The most widely used by researchers for pre-processing, among others, is tokenization, POS-tagging, stop word, punctuation, and stemming. After the pre-processing steps are completed, the data should be ready for the sarcasm classification phase.

TABLE II

CLASSIFICATION SCHEME OF SARCASM RESEARCH

\begin{tabular}{lll}
\hline \multicolumn{1}{c}{ Scheme } & \multicolumn{1}{c}{ Criteria } & \multicolumn{1}{c}{ References } \\
\hline Dataset & Twitter & {$[6],[14],[15]$} \\
& Online News & {$[14]$} \\
& Other & {$[16]-[18]$} \\
Pre- & Tokenization & {$[15],[11]$} \\
processing & POS Tagging & {$[19],[20],[9]$} \\
& Stop Word & {$[21]$} \\
& Punctuation & {$[20],[19]$} \\
& Stemming & {$[10],[21],[22]$} \\
Approach & Rule-based & {$[23],[24],[25]$} \\
& Semi- & {$[23],[6]$} \\
& Supervised & {$[20],[22]$} \\
Annotation & Manual & {$[20],[26],[27],[28],[29]$} \\
& Distant & {$[30],[31]$} \\
Feature & N-gram & {$[31],[20]$} \\
& Sentiment & {$[25],[15],[32],[33],[34],[33],[35]$} \\
& Pragmatic & {$[33],[26],[36],[16]$} \\
Context & Pattern & {$[20],[27],[27],[27]$} \\
& Author & {$[37],[38],[39]$} \\
& Conversation & {$[40],[41],[39],[42],[18]$} \\
Method & Naive Bayes & {$[43],[36],[44],[35],[45],[46],[47]$} \\
& SVM & {$[40],[44],[35],[48],[49],[50],[51],[52]$} \\
& KNN & {$[53],[49]$} \\
& CNN & {$[54],[55],[56]$} \\
\hline
\end{tabular}


3) Approach: A variety of methods have been done with many varieties of techniques, including statistical models, sentiment analysis, pattern recognition, supervised or unsupervised machine learning [57]. The supervised and semi-supervised approach is used for building a model to classify data through a statistical and logical process. Meanwhile, the pattern-based method uses semantic, syntactic, and stylistic properties of sentences in any language such as phrase pattern, lexical, and structural properties to analyse the value of the sentence.

4) Annotation: Manual annotation means each tweet is labelled by the annotators to decide whether the statement of the tweet is recognized as sarcasm sentences or not. The distant technique to create datasets is the use of hashtag-based supervision. Many approaches use hashtags in tweets as indicators of sarcasm, such as \#sarcasm or \#sarcastic. Sarcasm sentences found within those hashtags can be detected more efficiently [58].

5) Feature: The lexical feature is based on n-grams that occur more than twice in the training data, while the pragmatic feature determines when the sentiment of the sentence differs from the emoticons or smiles [59]. NGram model is probabilistic of words to predict the next item in a sequence with (n-1) order Markov Model. The prediction could be made based on a single preceding item $\mathrm{N}$-gram. Items that are a part of the speech of the words used in the sentences then classified using trigrams as features for the model and not suggest the higher n-grams, because its ineffectively in classification [60].

6) Context: The existing work currently focuses on utilizing contextual information in the sentences (e.g., a conversation or the history of the target author). Contextaugmented neural models can effectively decode sarcastic clues from contextual information, and give a relative improvement in the detection performance. Including the contextual features will significantly improve the performance, and that the most significant gains are attributable to features encoding information about the authors of tweets [56].

7) Method: The method is the approach that is used for sarcasm detection through specific evidence. The used models vary, such as Naive Bayes, SVM, KNN, and CNN. CNN has been a target of attention in social media because has shown excellent capabilities for modelling complex word composition in a sentence [54]. CNN is generally used in computer vision, however recently
CNN has been applied to various NLP tasks such as sarcasm detection and the results were promising, as we can see in Table III. The accuracy using skip-gram and sentiment until character level gives over than $92 \%$ and 92\% for F-score using bag-of-words classification method. The range of accuracy values is wide enough, between 54\% until $90 \%$. These results can be a challenge in finding the most appropriate features and classification techniques for the best way to detect sarcasm. The features performance for various machine learning algorithms that have been used as classifiers, as well as SVM, K-NN, random forest, NB, and CNN. The classification techniques are still dominated by SVM and KNN models, giving the highest performance accuracy value for sarcasm detection, above $80 \%$. The highest precision values above $72 \%$ are generated using SVM and KNN.

The classification methods are chosen depending on the task or a particular problem to be solved. It has been recommended to consider as many algorithms as possible to determine the validity of the proposed process, include pre-processing. The language most widely used by researchers in English and others that are used, but not many are Portuguese, Dutch, Chinese, India, and Indonesian. Sarcasm detection studies involving Indonesian-language social media have been carried out [7] using negative word count information and the number of interjection words. This study uses SentiWordNet for the sentiment classification process and produces an accuracy value under $60 \%$. A review study conducted in 2010 said that the value of accuracy and precision obtained when detecting sarcasm, which is higher than $90 \%$, was carried out using the Supervised Sarcasm Identification feature. This research was conducted for Twitter and Amazon data with Englishlanguage texts [51].

Table IV shows the most relevant studies regarding sarcasm detection with the highest number of citations and the most topics discussed in the articles. Detection of sarcasm is indeed a topic that closely concerned with machine learning; therefore, most of the articles discussing the use of machine learning to solve the problems of sarcasm detection. The other issues are still dominated by sentiment analysis, features, social media, and the algorithm. Meanwhile, natural language processing, neural network, and deep learning for sarcasm detection research are most discussed in the last three years, as mention by [9]. 
TABLE III

FEATURES PERFORMANCE

\begin{tabular}{clccc}
\hline Ref. & \multicolumn{1}{c}{ Features } & Accuracy (\%) & Classification Technique & Language \\
\hline$[61]$ & Emoticon, Lexicon, Syntactic, Semantic, Unigram & 60 & Multi-Class & English \\
{$[43]$} & N-gram, Skip-gram. & 65 & NB & English \\
{$[62]$} & Lexical, Pragmatic, Emoticon. & 71 & SVM & Dutch \\
{$[7]$} & Unigram, Negativity, Interjection & 53 & NB & Indonesia \\
{$[63]$} & Semantic, Expression, Emoticon. & 54 & SVM & English \\
{$[64]$} & N-gram, Emoticon, Interjection & $\mathbf{8 3}$ & SVM & English \\
{$[20]$} & Emoticon, Interjection, Syntactic-Semantic. & 86 & SVM & SVM \\
{$[65]$} & Skip-gram (word2vec) & 80 & KNN & English \\
{$[39]$} & Conversation context & $\mathbf{9 0}$ & CNN & English \\
{$[66]$} & Sentiment (+) (-) Character level & 54 & CNN & English \\
{$[67]$} & Positive Verbs \& Negative Situation & $\mathbf{9 5}$ & SVM & English \\
\hline
\end{tabular}

TABLE IV

SARCASM DETECTION RESEARCH CHALENGES

\begin{tabular}{cll}
\hline Ref. & \multicolumn{1}{c}{ State of the Art } & \multicolumn{1}{c}{ Future Research Challenges } \\
\hline$[60]$ & $\begin{array}{l}\text { Hashtag \#sarcasm using true-positive and false- } \\
\text { positive rate }\end{array}$ & $\begin{array}{l}\text { Different language and more subtle variants like } \\
\text { understatements, euphemisms, and litotes }\end{array}$ \\
{$[36]$} & $\begin{array}{l}\text { SVM and Decision Tree modelling for context } \\
\text { incongruity }\end{array}$ & $\begin{array}{l}\text { Use other data type, dialogue systems, or review ranking } \\
\text { systems }\end{array}$ \\
{$[34]$} & The context-based pattern in Hindi Tweets & $\begin{array}{l}\text { Sufficient dataset and language for training and testing } \\
\text { A large number of data compare human annotator with } \\
\text { machine annotator and emoticon processing }\end{array}$ \\
{$[46]$} & Classifier model with some features & $\begin{array}{l}\text { Full thread context and utilize external background } \\
\text { knowledge to model the sentiment }\end{array}$ \\
{$[41]$} & Conversation context modelling using LSTM & $\begin{array}{l}\text { Improve the model with more context } \\
\text { Incorporating the current method as a set of features for a } \\
\text { statistical classifier }\end{array}$ \\
{$[39]$} & Context-augmented CNN & $\begin{array}{l}\text { Expand the dataset and the method } \\
\text { RNN model, other features, word intensifier, punctuations, } \\
\text { and deep learning hyper-parameter optimizing }\end{array}$ \\
\hline 23$]$ & Context2Vec using Bidirectional LSTM &
\end{tabular}

Fig. 5 presents a maps of keywords which is calculated based on co-occurrence data that collect from title and abstract of all the articles. There are two visualizations shown, that are: network visualization and density visualization. The network visualization, the colors indicate by a label and, by a circle. The more important an item, the larger its label and its circle. The network visualization shows the cluster to which an article was assigned by the clustering technique used by
VOSviewer. There is a strong connection between the word "sarcasm" with "sarcasm detection", "approach", "tweet", and "feature". Meanwhile, density visualization can be used to see parts of research that are still rarely done. There are strong similarities that result between the clustering obtained from VOSviewer and our classification technique above. The three clusters that correspond strongly with our manual classification are sarcasm detection, approach, and context. 




(a)

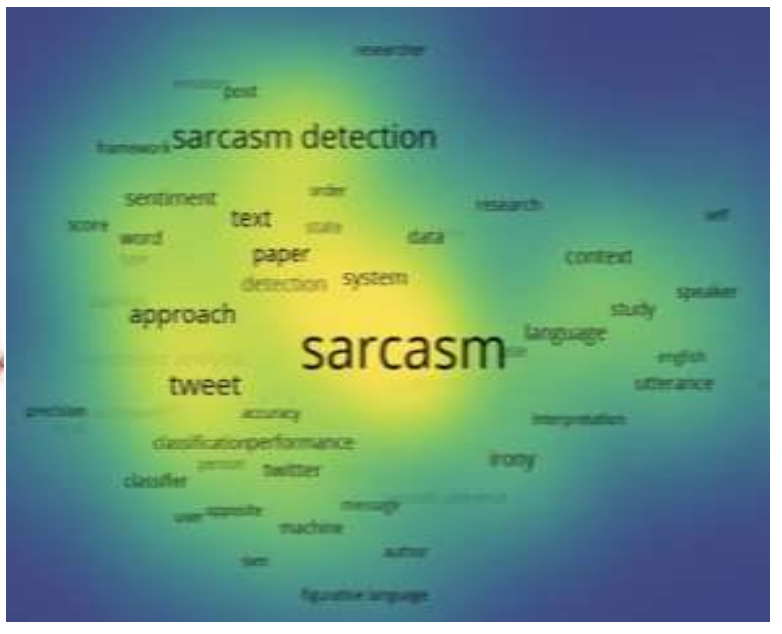

(b)

Fig. 5 Network (a) and density (b) visualization for sarcasm keyword

\section{CONCLUSIONS}

Various machine learning algorithm has been used for sarcasm classifiers, such as SVM, K-NN, NB, and NN. In general, there are extensive choices of which classification method can be used when designing a sarcasm with large and unstructured data. Text dataset, with noisy and unstructured data, can be successfully classified using a deep learning approach such as CNN or RNN. CNN uses a variation of multilayer perceptron designed to reduce pre-processing. RNN with LSTM (Long Short Term Memory) and GRU (Gated Recurrent Unit) can be used for prediction to the sequence of texts. LSTM method with an attention mechanism model to detect English ironic sentences from Twitter shows a better performance with a combination of CNN, LSTM, and Deep Neural Network.

This classification scheme study reveals extensive guidance for other academics, researchers, and practitioners that are researching the field of sarcasm detection and recognition. This review will give a further understanding of various concepts about sarcasm detection in social media. The results showed explosive growth in the number of sarcasm detection articles published. This study describes the increase of research benefit in sarcasm detection as necessary and legitimate research topics. The most important aspect of future work in sarcasm detection is dataset usage with various languages and contextual information that effectively detect sarcasm and give an improvement in the detection performance. Furthermore, we believe that there are some prospective hybrid approaches for classification and feature extraction to identify the sarcasm sentence using deep learning with big data. Combining different methods between machine learning and deep learning to facilitate sarcasm patterns and recognition is possible to do and developing contextual information of dataset to improve the performance of sarcasm detection.

\section{REFERENCES}

[1] H. S. Cheang and M. D. Pell, "The sound of sarcasm," Speech Commun., vol. 50, no. 5, pp. 366-381, May 2008.

[2] A. T. Martini, M. Farrukh, and H. Ge, "Recognition of ironic sentences in twitter using attention-based LSTM," Int. J. Adv. Comput. Sci. Appl., vol. 9, no. 8, pp. 7-11, 2018.

[3] D. Nozza, E. Fersini, and E. Messina, "Unsupervised Irony Detection: A Probabilistic Model with Word Embeddings," in Proceedings of the 8th International Joint Conference on Knowledge Discovery, Knowledge Engineering and Knowledge Management, 2016, vol. 1, pp. 68-76.

[4] A. Musolff, "Metaphor, irony and sarcasm in public discourse," J. Pragmat., vol. 109, pp. 95-104, Feb. 2017.

[5] D. Thompson and R. Filik, "Sarcasm in Written Communication: Emoticons are Efficient Markers of Intention," J. Comput. Commun., vol. 21, no. 2, pp. 105120, Mar. 2016.

[6] L. Peled and R. Reichart, "Sarcasm SIGN: Interpreting Sarcasm with Sentiment Based Monolingual Machine Translation," in Proceedings of the 55th Annual Meeting of the Association for Computational Linguistics (Volume 1: Long Papers), 2017, vol. 1, pp. 1690-1700.

[7] E. Lunando and A. Purwarianti, "Indonesian social media sentiment analysis with sarcasm detection," 2013 Int. Conf. Adv. Comput. Sci. Inf. Syst. ICACSIS 2013, pp. 195-198, Sep. 2013.

[8] A. Culotta, "Towards detecting influenza epidemics by analyzing Twitter messages," in Proceedings of the First Workshop on Social Media Analytics - SOMA '10, 2010, 
no. 4 , pp. $115-122$.

[9] S. G. Wicana, T. Y. Ibisoglu, and U. Yavanoglu, "A Review on Sarcasm Detection from Machine-Learning Perspective," in 2017 IEEE 11th International Conference on Semantic Computing (ICSC), 2017, pp. 469-476.

[10] S. K. Bharti, K. Sathya Babu, and S. K. Jena, "Harnessing Online News for Sarcasm Detection in Hindi Tweets," in Lecture Notes in Computer Science (including subseries Lecture Notes in Artificial Intelligence and Lecture Notes in Bioinformatics), vol. 10597 LNCS, 2017, pp. 679-686.

[11] M. Y. Manohar and P. Kulkarni, "Improvement sarcasm analysis using NLP and corpus based approach," Proc. 2017 Int. Conf. Intell. Comput. Control Syst. ICICCS 2017, vol. 2018-Janua, pp. 618-622, Jun. 2018.

[12] R. Afiyati, E. Winarko, and A. Cherid, "Recognizing the sarcastic statement on WhatsApp Group with Indonesian language text," in 2017 International Conference on Broadband Communication, Wireless Sensors and Powering, BCWSP 2017, 2018, vol. 2018-Janua, pp. 16.

[13] A. G. Prasad, S. Sanjana, S. M. Bhat, and B. S. Harish, "Sentiment analysis for sarcasm detection on streaming short text data," in 2017 2nd International Conference on Knowledge Engineering and Applications (ICKEA), 2017, vol. 2017-Janua, no. 2009, pp. 1-5.

[14] R. Justo, J. M. Alcaide, M. I. Torres, and M. Walker, "Detection of Sarcasm and Nastiness: New Resources for Spanish Language," Cognit. Comput., vol. 10, no. 6, pp. 1135-1151, Dec. 2018.

[15] J. G. C. M. Ræder and B. Gambäck, "Sarcasm Annotation and Detection in Tweets," in Lecture Notes in Computer Science (including subseries Lecture Notes in Artificial Intelligence and Lecture Notes in Bioinformatics), vol. 10762 LNCS, 2018, pp. 58-70.

[16] M. S. M. Suhaimin, M. H. A. Hijazi, R. Alfred, and F. Coenen, "Natural language processing based features for sarcasm detection: An investigation using bilingual social media texts," ICIT 2017 - 8th Int. Conf. Inf. Technol. Proc., pp. 703-709, May 2017.

[17] D. Das and A. J. Clark, "Sarcasm detection on facebook: A supervised learning approach," Proc. 20th Int. Conf. Multimodal Interact. ICMI 2018, no. October, 2018.

[18] S. Lukin and M. Walker, "Really? Well. Apparently Bootstrapping Improves the Performance of Sarcasm and Nastiness Classifiers for Online Dialogue," Proc. Work. Lan- guage Anal. Soc. Media, vol. 1, pp. 30-40, Aug. 2017.

[19] P. Bhattacharyya, "Sarcasm Detection : A Computational and Cognitive Study," 2018.

[20] M. Bouazizi, T. Otsuki Ohtsuki, and T. Otsuki, "A Pattern-Based Approach for Sarcasm Detection on Twitter," IEEE Access, vol. 4, pp. 5477-5488, 2016.
[21] K. Ravi and V. Ravi, "A novel automatic satire and irony detection using ensembled feature selection and data mining," Knowledge-Based Syst., vol. 120, pp. 15-33, Mar. 2017.

[22] A. Rajadesingan, "Detecting Sarcasm on Twitter: A Behavior Modeling Approach," Proc. Eighth ACM Int. Conf. Web Search Data Min., vol. Proceeding, no. September, pp. 97-106, 2014.

[23] A. Joshi, S. Agrawal, P. Bhattacharyya, and M. J. Carman, "Expect the Unexpected: Harnessing Sentence Completion for Sarcasm Detection," in Communications in Computer and Information Science, vol. 781, 2018, pp. 275-287.

[24] D. Ghosh, W. Guo, and S. Muresan, "Sarcastic or Not: Word Embeddings to Predict the Literal or Sarcastic Meaning of Words," in Proceedings of the 2015 Conference on Empirical Methods in Natural Language Processing, 2015, no. September, pp. 1003-1012.

[25] A. Mishra, D. Kanojia, and P. Bhattacharyya, "Predicting Readers' Sarcasm Understandability by Modeling Gaze Behavior," Proc. 30th Conf. Artif. Intell. (AAAI 2016), pp. 3747-3753, 2016

[26] L. H. Son, A. Kumar, S. R. Sangwan, A. Arora, A. Nayyar, and M. Abdel-Basset, "Sarcasm detection using soft attention-based bidirectional long short-term memory model with convolution network," IEEE Access, vol. 7, pp. 23319-23328, 2019.

[27] S. M. Basha and D. S. Rajput, "Parsing Based Sarcasm Detection from Literal Language in Tweets," Recent Patents Comput. Sci., vol. 11, no. 1, pp. 62-69, 2018.

[28] A. Joshi, P. Bhattacharyya, and M. J. Carman, "Automatic Sarcasm Detection," ACM Comput. Surv., vol. 50, no. 5, pp. 1-22, Sep. 2017.

[29] F. Kunneman, C. Liebrecht, M. van Mulken, and A. van den Bosch, "Signaling sarcasm: From hyperbole to hashtag," Inf. Process. Manag., vol. 51, no. 4, pp. 500 509, Jul. 2015.

[30] A. Agrawal and A. An, "Affective representations for sarcasm detection," in 41st International ACM SIGIR Conference on Research and Development in Information Retrieval, SIGIR 2018, 2018, pp. 10291032.

[31] A. Mishra, D. Kanojia, S. Nagar, K. Dey, and P. Bhattacharyya, "Harnessing Cognitive Features for Sarcasm Detection,” pp. 1095-1104, 2017.

[32] M. Bouazizi and T. Ohtsuki, "Sarcasm detection in twitter: »all your products are incredibly amazing!!!» are they really?," in 2015 IEEE Global Communications Conference, GLOBECOM 2015, 2015.

[33] E. Fersini, F. A. Pozzi, and E. Messina, "Detecting irony and sarcasm in microblogs: The role of expressive signals and ensemble classifiers," in Proceedings of the 2015 IEEE International Conference on Data Science and Advanced Analytics, DSAA 2015, 2015, pp. 1-8. 
[34] S. K. Bharti, K. S. Babu, and R. Raman, "Context-based Sarcasm Detection in Hindi TweetsTechnology," 2017 9th Int. Conf. Adv. Pattern Recognition, ICAPR 2017, no. December, pp. 410-415, 2017.

[35] J. M. Alcaide, R. Justo, and M. I. Torres, "Combining Statistical and Semantic Knowledge for Sarcasm Detection in Online Dialogues," in Lecture Notes in Computer Science (including subseries Lecture Notes in Artificial Intelligence and Lecture Notes in Bioinformatics), vol. 9117, 2015, pp. 662-671.

[36] S. Mukherjee and P. K. Bala, "Detecting sarcasm in customer tweets: An NLP based approach," Ind. Manag. Data Syst., vol. 117, no. 6, pp. 1109-1126, Jul. 2017.

[37] A. Khattri, A. Joshi, P. Bhattacharyya, and M. Carman, "Your Sentiment Precedes You: Using an author's historical tweets to predict sarcasm," in Proceedings of the 6th Workshop on Computational Approaches to Subjectivity, Sentiment and Social Media Analysis, 2015, no. 2013, pp. 25-30.

[38] Y. Alex Kolchinski and C. Potts, "Representing social media users for sarcasm detection," in Proceedings of the 2018 Conference on Empirical Methods in Natural Language Processing, EMNLP 2018, 2020.

[39] Y. Ren, D. Ji, and H. Ren, "Context-augmented convolutional neural networks for twitter sarcasm detection," Neurocomputing, vol. 308, pp. 1-7, Sep. 2018.

[40] Z. Wang, Z. Wu, R. Wang, and Y. Ren, "Twitter Sarcasm Detection Exploiting a Context-Based Model," in Lecture Notes in Computer Science (including subseries Lecture Notes in Artificial Intelligence and Lecture Notes in Bioinformatics), vol. 9418, 2015, pp. 77-91.

[41] D. Ghosh, A. R. Fabbri, and S. Muresan, "Sarcasm analysis using conversation context," Comput. Linguist., vol. 44, no. 4, pp. 755-792, 2018.

[42] G. Abercrombie and D. Hovy, "Putting Sarcasm Detection into Context: The Effects of Class Imbalance and Manual Labelling on Supervised Machine Classification of Twitter Conversations," in Proceedings of the ACL 2016 Student Research Workshop, 2016, pp. 107-113.

[43] S. Mukherjee and P. K. Bala, "Sarcasm detection in microblogs using Naïve Bayes and fuzzy clustering," Technol. Soc., vol. 48, pp. 19-27, Feb. 2017.

[44] S. K. Bharti, R. Naidu, and K. S. Babu, "Hyperbolic Feature-based Sarcasm Detection in Tweets: A Machine Learning Approach," in 2017 14th IEEE India Council International Conference, INDICON 2017, 2017, no. December, pp. 1-6.

[45] A. Onan, "Sarcasm Identification on Twitter: A Machine Learning Approach," in Advances in Intelligent Systems and Computing, vol. 573, 2017, pp. 374-383.

[46] D. Al-Ghadhban, E. Alnkhilan, L. Tatwany, and M. Alrazgan, "Arabic sarcasm detection in Twitter," in
Proceedings - 2017 International Conference on Engineering and MIS, ICEMIS 2017, 2018, vol. 2018Janua, pp. 1-7.

[47] N. Bhan and M. D'silva, "Sarcasmometer using sentiment analysis and topic modeling," in 2017 International Conference on Advances in Computing, Communication and Control (ICAC3), 2017, vol. 2018Janua, pp. 1-7.

[48] K. Sreelakshmi and P. C. Rafeeque, "An Effective Approach for Detection of Sarcasm in Tweets," 2018 Int. CET Conf. Control. Commun. Comput. IC4 2018, pp. 377-382, 2018.

[49] P. D. Manjusha and C. Raseek, "Convolutional neural network based simile classification system," 2018 Int. Conf. Emerg. Trends Innov. Eng. Technol. Res. ICETIETR 2018, pp. 1-5, 2018.

[50] P. Tungthamthiti, "Recognition of sarcasm in tweets based on concept level sentiment analysis and supervised learning approaches," in Proceedings of Pacific, aclweb, 2014, pp. 404-413.

[51] A. D. Dave and N. P. Desai, "A comprehensive study of classification techniques for sarcasm detection on textual data," in 2016 International Conference on Electrical, Electronics, and Optimization Techniques (ICEEOT), 2016, pp. 1985-1991.

[52] D. Ghosh, W. Guo, and S. Muresan, "Sarcastic or Not: Word Embeddings to Predict the Literal or Sarcastic Meaning of Words," Proc. 2015 Conf. Empir. Methods Nat. Lang. Process., no. September, pp. 1003-1012, 2015.

[53] M. Khachidze, M. Tsintsadze, M. Archuadze, G. Besiashvili, and I. Javakhishvili, "Concept Pattern Based Text Classification System Development for Georgian Text Based Information Retrieval," Balt. J. Mod. Comput., vol. 3, no. 4, pp. 307-317, 2015.

[54] A. Ghosh and D. T. Veale, "Fracking Sarcasm using Neural Network," in Proceedings of the 7th Workshop on Computational Approaches to Subjectivity, Sentiment and Social Media Analysis, 2016, no. May, pp. 161-169.

[55] R. Schifanella, P. de Juan, J. Tetreault, and L. Cao, "Detecting Sarcasm in Multimodal Social Platforms," in Proceedings of the 2016 ACM on Multimedia Conference - MM '16, 2016, pp. 1136-1145.

[56] S. Amir, B. C. Wallace, H. Lyu, and P. C. M. J. Silva, "Modelling Context with User Embeddings for Sarcasm Detection in Social Media," Jul. 2016.

[57] P. Tungthamthiti, "Recognition of sarcasm in tweets based on concept level sentiment analysis and supervised learning approaches," in Proceedings of Pacific, aclweb, 2014, pp. 404-413.

[58] D. Maynard and M. a. Greenwood, "Who cares about Sarcastic Tweets? Investigating the Impact of Sarcasm on Sentiment Analysis.," Lr. 2014 Proceedings. Lang. Resour. Eval. Conf., vol. Proceeding, pp. 4238-4243, 2014. 
[59] P. Nagwanshi and C. E. Veni Madhavan, "Sarcasm Detection using Sentiment and Semantic Features," in Proceedings of the International Conference on Knowledge Discovery and Information Retrieval, 2014, pp. 418-424.

[60] C. Liebrecht, F. Kunneman, and A. Van Den Bosch, "The perfect solution for detecting sarcasm in tweets \# not," Proc. ofthe 4th Work. Comput. Approaches to Subj. Sentim. Soc. Media Anal., pp. 29-37, 2013.

[61] M. Bouazizi and T. Ohtsuki, "A Pattern-Based Approach for Multi-Class Sentiment Analysis in Twitter," IEEE Access, vol. 5, pp. 20617-20639, 2017.

[62] R. González-Ibáñez, S. Muresan, and N. Wacholder, "Identifying Sarcasm in Twitter: A Closer Look," Proc. 49th Annu. Meet. Assoc. Comput. Linguist. Hum. Lang. Technol. short Pap. 2, no. 2010, pp. 581-586, 2011.

[63] A. Rajadesingan, R. Zafarani, and H. Liu, "Sarcasm Detection on Twitter," in Proceedings of the Eighth ACM International Conference on Web Search and Data Mining - WSDM '15, 2015, pp. 97-106.

[64] J. Ling and R. Klinger, “An Empirical, Quantitative
Analysis of the Differences Between Sarcasm and Irony," in Lecture Notes in Computer Science (including subseries Lecture Notes in Artificial Intelligence and Lecture Notes in Bioinformatics), vol. 9989 LNCS, 2016, pp. 203-216.

[65] P. Mehndiratta, S. Sachdeva, D. Soni, S. Sachdevai, and D. Soni, "Detection of Sarcasm in Text Data using Deep Convolutional Neural Networks," Scalable Comput. Pract. Exp., vol. 18, no. 3, pp. 821-824, Sep. 2017.

[66] M. Arora and V. Kansal, "Character level embedding with deep convolutional neural network for text normalization of unstructured data for Twitter sentiment analysis," Soc. Netw. Anal. Min., vol. 9, no. 1, p. 0, 2019.

[67] E. Riloff et al., "Sarcasm as Contrast between a Positive Sentiment and Negative Situation," EMNLP 2013 - 2013 Conf. Empir. Methods Nat. Lang. Process. Proc. Conf., no. Emnlp, pp. 704-714, 2013.

[68] J. Tarigan and A. S. Girsang, "Word similarity score as augmented feature in sarcasm detection using deep learning," Int. J. Adv. Comput. Res., vol. 8, no. 39, pp. 354-363, 2018. 